\title{
Layer-by-Layer Arrangement by Protein-Protein Interaction of Sulfite Oxidase and Cytochrome $c$ Catalyzing Oxidation of Sulfite
}

\author{
Roman Dronov, Dirk G. Kurth, Helmuth Möhwald, Roberto Spricigo, Silke Leimkühler, Ulla \\ Wollenberger, K.V. Rajagopalan, Frieder W. Scheller, and Fred Lisdat
}

\section{Supporting information}

\section{Experimental}

Materials. Human SOx (EC 1.8.3.1) was produced by expression in E.coli cells and purified by affinity chromatography (Temple, C. A.; Graf, T. N.; Rajagopalan, K.V. Arch Biochem Biophys , 2000, 383(2), 281-287). Horse-heart cyt.c was purchased from Sigma (Steinheim, Germany). 11-mercapto-1undecanol (MU) and 11-mercapto-1-undecanoic acid (MUA) were provided by Aldrich (Taufkirchen, Germany). Sulfuric acid, dipotassium hydrogen phosphate, potassium dihydrogen phosphate and sodium sulfite were supplied by Merck (Darmstadt, Germany). 0.5 mm diameter Au wires were purchased from Goodfellow (Bad Nauheim, Germany). All solutions were prepared in $18 \mathrm{M} \Omega$ Millipore water (Eschborn, Germany).

Buffers. Phosphate buffers were prepared using potassium dihydrogen phosphate and dipotassium hydrogen phosphate with $\mathrm{pH}$ adjustment by potassium hydroxide or phosphoric acid. Buffer 1: $5 \mathrm{mM}$ phosphate $\mathrm{pH} 7.0$ was used for cyt.c monolayer assembly and recording of cyclic voltammograms. Buffer 2: $0.5 \mathrm{mM}$ phosphate $\mathrm{pH} 5.0$ was used for build up of SOx/cyt.c assembly.

Apparatus. Electrochemical experiments were carried out in a $1 \mathrm{ml}$ cell using a $\mathrm{Ag} / \mathrm{AgCl} / 1 \mathrm{M} \mathrm{KCl}$ reference electrode (Biometra, Germany) and a Pt-wire counter electrode. The working electrodes were modified gold wires. Cyclic voltammograms were recorded using an Autolab System (Metrohm, The Netherlands). QCM experiments were performed at the piezoelectric detector Multilab 3900 obtained from J. Kitlička (Brno, Czech Republic) using gold covered (5 mm in diameter) quartz crystals (ICMFG Oklahoma, USA).

Electrode preparation. SOx/cyt.c arrangement was prepared by alternating incubation of a cyt.c monolayer electrode into (i) a solution containing $10 \mu \mathrm{M}$ SOx and $1 \mu \mathrm{M}$ cyt.c for $15 \mathrm{~min}$, followed by a washing step in buffer 2 , and (ii) a pure $20 \mu \mathrm{M}$ cyt.c solution for 10 min. The cyt.c monolayer was produced by $2 \mathrm{~h}$ adsorption of cyt.c (in buffer 1 ) onto a MUA/MU (1:3) pre-modified electrodes. The electrodes can be used at least one day after preparation.

Cyclic voltammetry. Electrochemical response of the electrodes was controlled by recording cyclic voltammograms (CV). After formation of the cyt.c monolayer, the protein surface concentration was checked by CV. During SOx/cyt.c assembly stages no CV measurements were performed. After the final assembly step the electrode was rinsed subsequently in buffer 2, buffer 1 , and placed into the measuring cell with immersion depth of $5 \mathrm{~mm}$. In the case of catalytic current recording, $10 \mu \mathrm{l}$ of sodium sulfite stock solution was added into the measuring cell and mixed with a pipette.

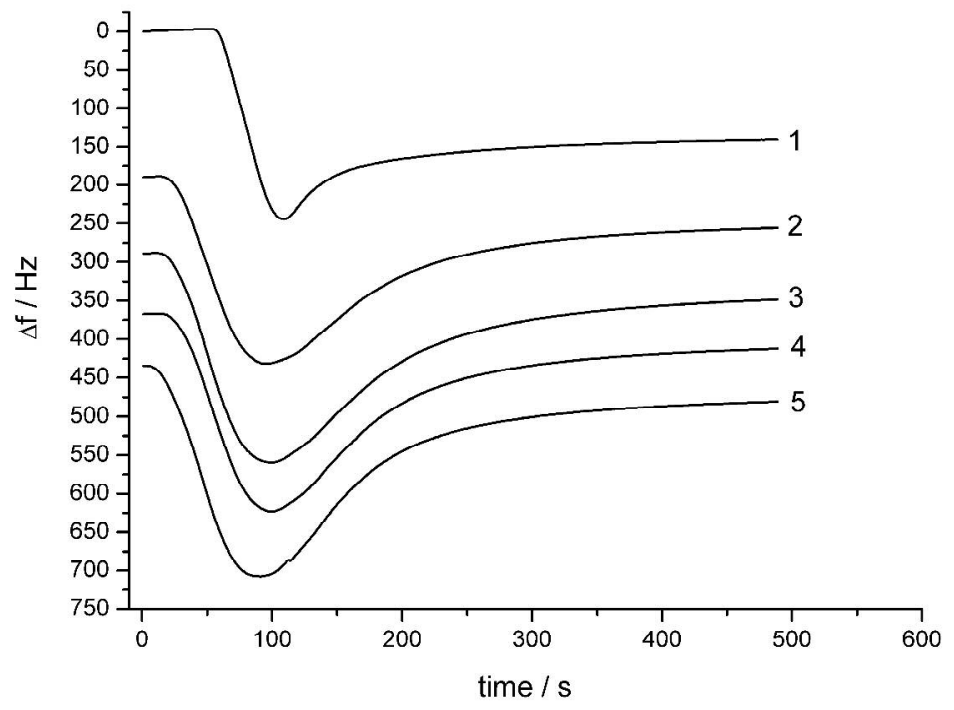

Figure S1. Deposition steps of SOx from a pure solution of $10 \mu \mathrm{M}$ concentration against $20 \mu \mathrm{M}$ cyt.c (not shown) measured by QCM. Numbers 1-5 denote adsorption cycles. 


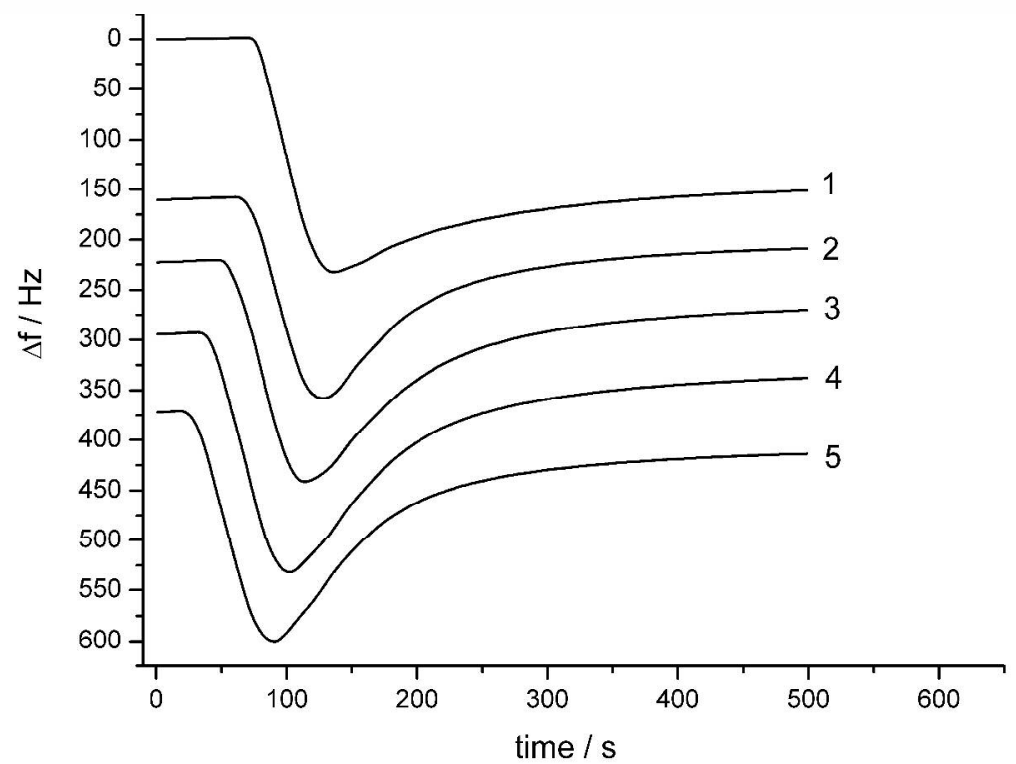

Figure S2. Deposition steps of SOx/cyt.c from a blended solution containing $10 \mu \mathrm{M}$ SOx and $1 \mu \mathrm{M}$ cyt.c against $20 \mu \mathrm{M}$ cyt.c (not shown) measured by QCM. Numbers 1-5 denote adsorption cycles.

The frequency decrease after injection corresponds to the mass deposit. The following increase after about 100 seconds can be understood as removal of loosely bound material.

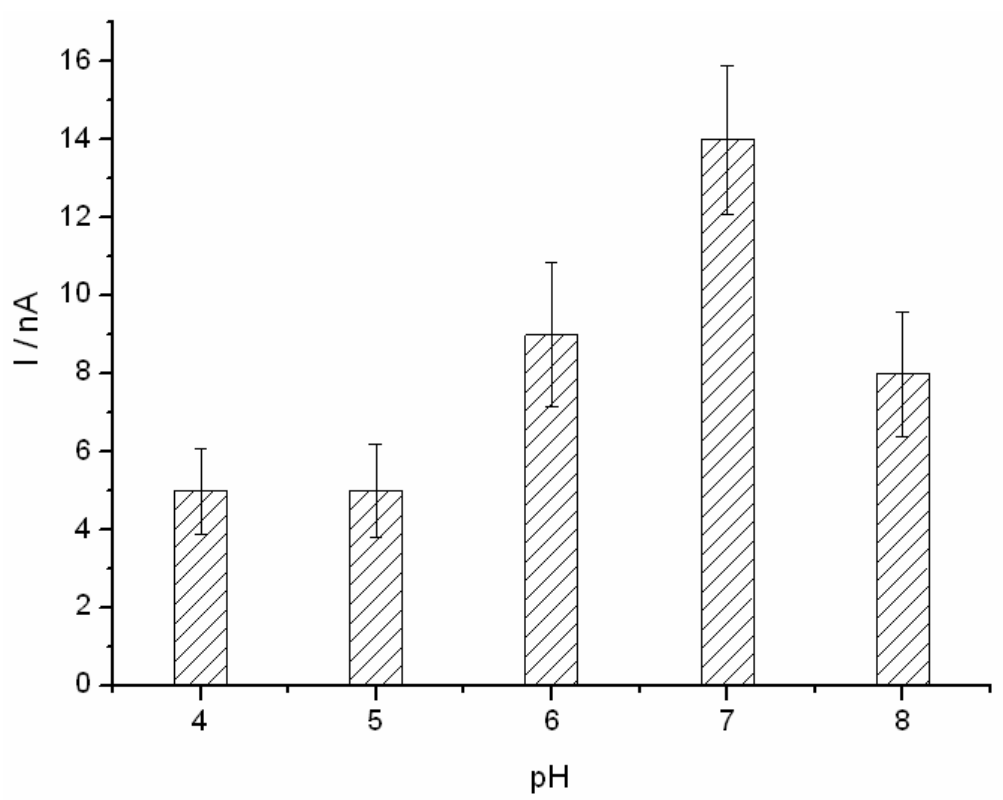

Figure S3. Catalytic current generated by Au-MUA/MU-\{(SOx/cyt.c-cyt.c) $\}_{3}-\mathrm{SOx} /$ cyt.c electrode at a potential of $+200 \mathrm{mV}$ at different $\mathrm{pH}$. Measurement performed at $5 \mathrm{mVs}^{-1}$ in presence of $1 \mathrm{mM}$ sulfite, error bars are derived from 2 measurements. 

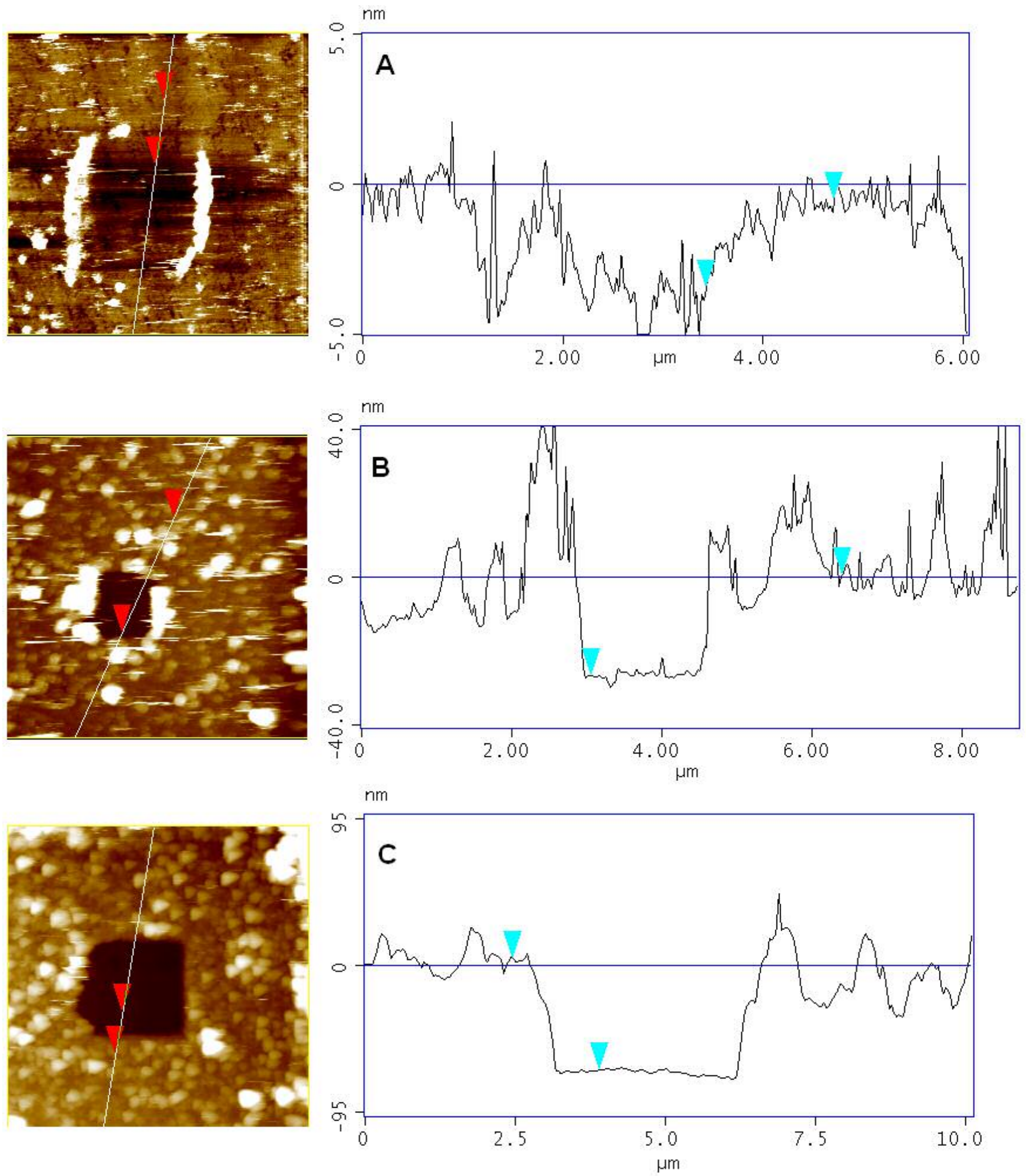

Figure S4. Topography images (left) and profile cross-sections (right) of Au-MUA/MU-cyt.c (A), Au-MUA/MU-cyt.c- $\{(\mathrm{SOx} / \mathrm{cyt} . c)-c y t . c\}_{3}$ (B), AuMUA/MU-cyt.c- $\{\text { (SOx/cyt.c)-cyt.c }\}_{6}(\mathrm{C})$. The measurements were carried out after removal of the material with the tip at a smaller scan size with a high force applied. The cross-section shows an increase in the film thickness upon consequent deposition steps. 